\title{
Polymer based Actuator for nozzle-diffuser Pumps in Plastic Microfluidic Systems
}

\author{
P. Sethu and C. H. Mastrangelo
}

\author{
Center for Wireless Integrated Microsystems \\ Department of Electrical Engineering and Computer Science \\ University of Michigan \\ Ann Arbor, MI 48109
}

\begin{abstract}
Plastic casting is an inexpensive technique for fabrication of disposable microfluidic systems for various biochemical applications. Pumps are critical for these systems and have a wide variety of applications including transport, mixing, cooling and creating pressure differences. In this paper we discuss the fabrication of a valve-less nozzle-diffuser pump integrated within a plastic microfluidic system. The pump uses thermal actuation, making use of the large volumetric expansion of a wax patch when it transitions from a solid to liquid phase. Testing of fabricated devices show maximum flow rates of $70 \mathrm{nl} / \mathrm{min}$ and generation of pressure heads of up to $1400 \mathrm{~Pa}$.
\end{abstract}

\section{INTRODUCTION}

Miniaturized systems for biochemical assays [1] significantly reduce cycle times, reagent costs and labor intensity. Plastic microfabrication techniques have been extensively used to build inexpensive analysis systems since most of these systems are targeted for single use applications. Commonly used techniques include injection molding, hot embossing and casting. Plastic casting has been shown to be an inexpensive technique capable of producing high-resolution features like channels and reservoirs that are required to construct these systems. Different materials like PDMS [2], Epoxies [3], Acrylics, etc. have been used for casting. We use epoxies to cast our systems since they provide a rigid platform and have high glass transition temperatures that allow for conventional photolithography to pattern electrodes and heaters.

Pumps in microfluidic systems are essential for fluid transport but also have other applications like mixing, cooling and pressure generation. Pumping is achieved using different actuation mechanisms like electrohydrodynamic, electrokinetic, mechanical displacement and bubble generation, which have all been miniaturized and used for pumping in glass and silicon based microsystems. Ideally we would like pumps to be inexpensive, simple to fabricate and easy to integrate within a plastic microfluidic system with high flow rates and pressure generation at relatively low power. For most bioassay applications bubble generation and local heating of the liquid are undesirable.

We have developed a wax-based actuator similar to the actuator developed by Carlen et. al. [4] that makes use of the large volumetric expansion of wax when it transitions from solid to liquid phase to generate large volume displacement that can be used to pump liquids. The fabrication technique is simple and inexpensive and these actuators can be mass fabricated on cast epoxy substrates. The actuator is used in a nozzle-diffuser [5] arrangement in series to generate a net fluid flow. The pump uses relatively low power and there is no bubble generation and operates at low temperatures $\left(<65^{\circ} \mathrm{C}\right.$ at the surface of the wax patch).

The device is fabricated using epoxy casting. The channel structure is cast on one substrate and the wax actuator is fabricated using a screen-printing technique on another substrate. Both the substrates are then bonded together using an adhesive bonding technique.

\section{THEORY}

Nozzle-diffuser pumps are built by coupling a nozzle, pumping chamber and diffuser together in series. The volume of fluid in the pumping chamber is periodically varied using an oscillating pressure (actuator). The kinetic energy of the fluid (velocity) is converted to potential energy (pressure) in both the nozzle and diffuser, but the efficiency of this process is much greater in the diffuser direction thus discharging more fluid through the diffuser. If $\mathrm{Q}_{\mathrm{d}}$ is the discharge through the diffuser and $\mathrm{Q}_{\mathrm{n}}$ the discharge through the nozzle then an increase in the chamber volume causes $\left|Q_{d}\right|>\left|Q_{n}\right|$ and a decrease in the chamber volume causes $\left|\mathrm{Q}_{\mathrm{d}}\right|<\left|\mathrm{Q}_{\mathrm{n}}\right|$ resulting in a net pumping action (Fig 1.).

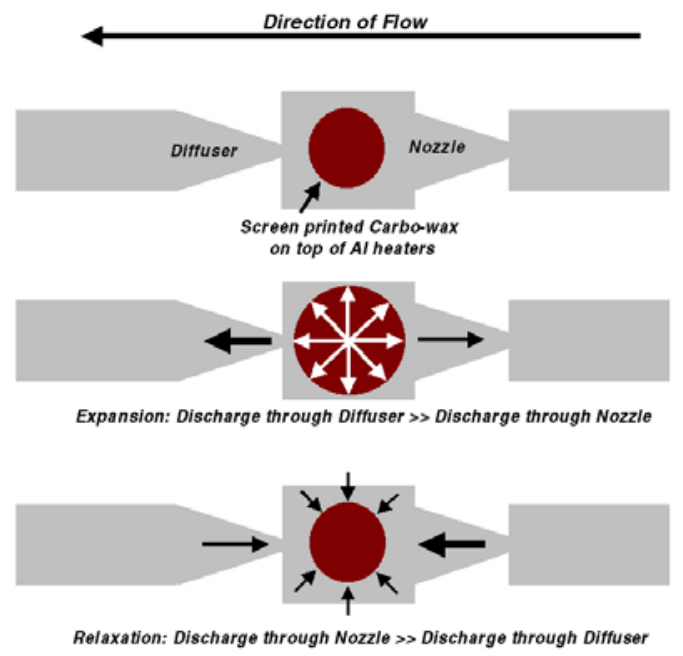

Figure 1. Principle of operation of nozzle-diffuser pumps and fluid flow with increasing and decreasing chamber volume

The pressure differences across the nozzle and diffuser are given by:

$$
\Delta \mathrm{P}_{\mathrm{d}}=\frac{\rho \mathrm{v}_{\mathrm{d}}{ }^{2} \xi_{\mathrm{d}}}{2} \text { and } \Delta \mathrm{P}_{\mathrm{n}}=\frac{\rho \mathrm{v}_{\mathrm{n}}{ }^{2} \xi_{\mathrm{n}}}{2}
$$


where:

$\Delta \mathrm{P}_{\mathrm{d}}$ and $\Delta \mathrm{P}_{\mathrm{n}}=$ Pressure difference across the diffuser and nozzle $\xi_{\mathrm{d}}$ and $\xi_{\mathrm{n}}=$ Pressure loss coefficients for the diffuser and nozzle $\mathrm{v}_{\mathrm{d}}$ and $\mathrm{v}_{\mathrm{n}}=$ Average velocities at the diffuser and nozzle

If $\xi_{\mathrm{d}}$ is less than $\xi_{\mathrm{n}}$ then pumping will occur in the direction of the diffuser [6]. These pumps cannot generate large pressure and are suitable for low-pressure fluidic systems.

And the volume flow rate is given by:

$$
\mathrm{Q}=2 \mathrm{~V} f \frac{\left[\left(\xi_{\mathrm{n}} / \xi_{\mathrm{d}}\right)^{1 / 2}-1\right]}{\left(\xi_{\mathrm{n}} / \xi_{\mathrm{d}}\right)^{1 / 2}+1}
$$

where:

$\mathrm{Q}=$ Volume flow rate

$\mathrm{V}=$ Volume variation per cycle

$f=$ pulse frequency

\section{FABRICATION}

The device is fabricated using plastic casting as shown in Figure 2. The substrate with the channels, reservoirs, pumping chamber and the nozzle-diffuser geometry is made using epoxy EPOTEK 301-2 FL (fig 2.a) which is a clear optical grade epoxy (>97\% light transmission), low viscosity (125 cpS at $100 \mathrm{rpm}$ ) and glass transition temperature $\mathrm{T}_{\mathrm{g}}=65^{\circ} \mathrm{C}$. A stamper with $50 \mu \mathrm{m}$ tall negative replicas of the channels, chamber and nozzle-diffuser geometry is formed by etching a silicon wafer using a deep RIE process $\left(\mathrm{SF}_{6} 160 \mathrm{sscm}, \mathrm{C}_{4} \mathrm{~F}_{8} 80 \mathrm{sscm} \mathrm{O} \mathrm{O}_{2} 13 \mathrm{sscm}\right.$, coil power $800 \mathrm{~W}$, platen power $600 \mathrm{~W})$. The stamper is then vapor deposited with a $1 \mu \mathrm{m}$ thick parylene layer, which serves as a release layer to aid separation of the cured plastic from the stamper. The silicon stamper is sandwiched with a Teflon O-ring ( $2 \mathrm{~mm}$ thick) and flat polypropylene disc in an aluminum mold. The epoxy resin is injected into the sandwich and maintained at $80^{\circ} \mathrm{C}$ for 3 hours and then the cured plastic is released. Access holes for channels and electrodes are drilled using a micro drill press

A second epoxy substrate is made by casting using EPOTEK -314 a clear, high temperature epoxy $\left(\mathrm{T}_{\mathrm{g}}=150^{\circ} \mathrm{C}\right)$. This epoxy is essential since metal heaters and contacts are patterned on the surface using conventional photolithography techniques and the plastic must be able to withstand temperatures of up to $110^{\circ} \mathrm{C}$. This substrate is made using the same technique but a blank silicon wafer with a $1 \mu \mathrm{m}$ thick parylene layer is used instead of the etched stamper. The released substrate is then vapor deposited with a $0.5 \mu \mathrm{m}$ thick parylene layer to improve adhesion of metals to the surface. Then metal layers $\mathrm{Cr} / \mathrm{Al}\left(500 \mathrm{~A} / 2000 \mathrm{~A}^{\circ}\right)$ are evaporated onto the substrate and heaters and contacts are patterned using photolithography.

The wax patches are made using a screen-printing process. The screen-printing process uses a $15 \mu \mathrm{m}$ thick stencil screen made of aluminum with precision cut openings for printing. The plastic substrate (EPOTEK 314) with the patterned heaters is then mounted on the screen printer and the openings on the screen and the heaters on the substrate are aligned and the screen is locked in position. The wax used is Poly ethylene Glycol, MW 8000 (PEG 8000, DOW Chemical Co.) which is a water-soluble carbowax (a)

Etched silicon negative replica of features

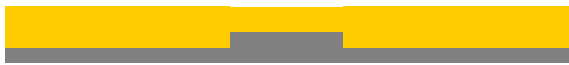

Cast plastic in molding setup

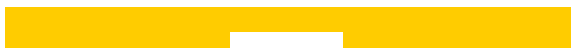

\section{Release plastic with micro-features}

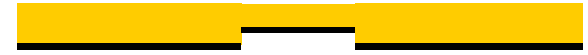

Spin on $0.5 \mu \mathrm{m}$ thick bonding wax solution

(b)

\section{Evaporate $\mathrm{Cr}$-Al layer on plastic substrate}

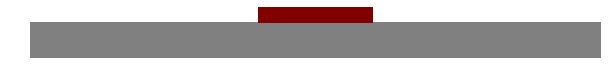

\section{Photo lithographically pattern heaters}

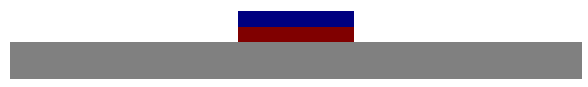

\section{Screen print Carbowax on top of heaters}

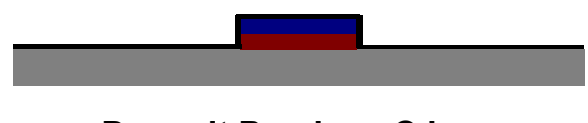

\section{Deposit Parylene C layer}

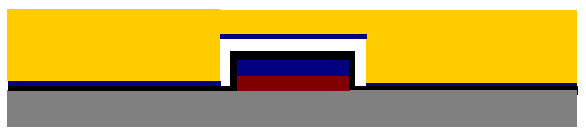

\section{Bond both plastic substrates together}

Figure 2 (a) and (b). Shown is the process flow for fabrication of the channel structure and wax actuator respectively. The two substrates are then bonded using an adhesive

with a melting point of $65^{\circ} \mathrm{C}$ and a volumetric expansion of $30 \%$ on transition from solid to liquid. The wax is melted by heating to $80{ }^{\circ} \mathrm{C}$ and then screen-printed using a teflon squeegee $(2 \mathrm{kPa}$ pressure, $8 \mathrm{ft} / \mathrm{min}$ speed). The substrate is released and placed in an oven at $65{ }^{\circ} \mathrm{C}$ for 3 minutes for the wax to re flow and to obtain a smooth surface. The contacts for the heaters are masked using 

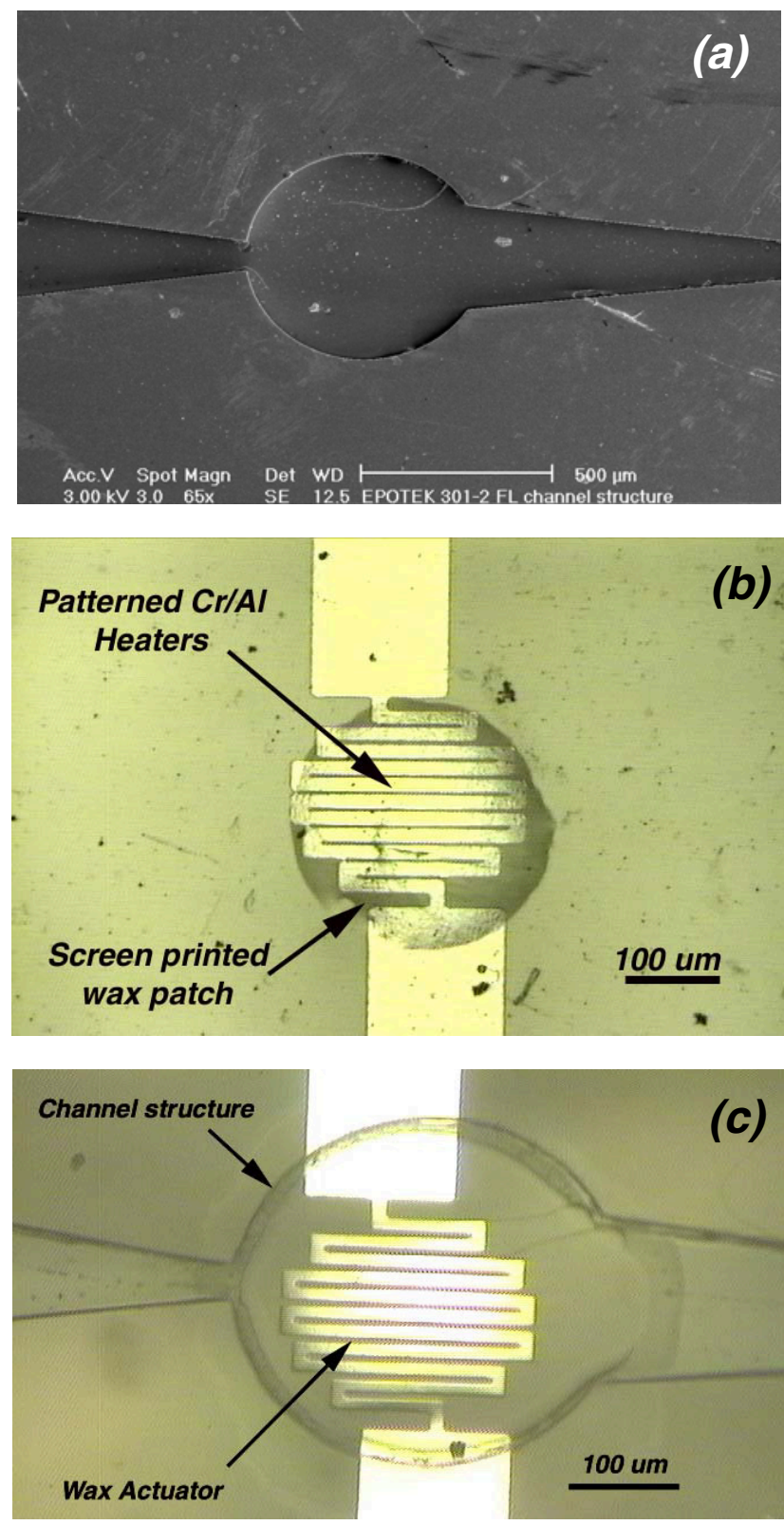

Figure 3. (a) Pictures show SEM of EPOTEK 301-2 FL substrate with chamber, channel and nozzle-diffuser geometry, (b) wax actuator on EPOTEK 314 substrate and (c) bonded nozzle-diffuser pump respectively.

masking tape and the substrate is coated with $4 \mu \mathrm{m}$ thick parylene $\mathrm{C}$ layer. Figure 3.b. shows a picture of $15 \mu \mathrm{m}$ tall wax actuator.

The EPOTEK 301-2 FL substrate with the channels and nozzle-diffuser geometry is spin coated with a $0.5 \mu \mathrm{m}$ thick layer of bonding wax solution (Blanchard bonding wax dissolved 1:1 by weight in acetone) and then aligned and bonded to the EPOTEK 314 substrate with the wax actuator using a laminator with the rollers at $60{ }^{\circ} \mathrm{C}$, speed of $2 \mathrm{ft} / \mathrm{min}$ and gauge of $4 \mathrm{~mm}$. The access holes for the electrodes are filled with conductive epoxy and cured at room temperature for 18 hours. Figure 3.c. shows a picture of the bonded device. The pumping chamber is $500 \mu \mathrm{m}$ in diameter with diffuser angle of $30^{\circ}$ and channel widths of $400 \mu \mathrm{m}$.

\section{EXPERIMENTAL DETAILS}

Tests were performed first on the fabricated wax actuator to obtain the deflection heights under different applied voltages. Individual dies with the fabricated actuator were wire bonded to a PC board and the PC board was setup under a ZYGO optical surface profilometer. The surface of the actuator corresponding to the different applied voltages was imaged.

Experiments were also performed to calculate the flow rate and pumping pressure. Flow rate calculations were performed by introducing $3 \mu \mathrm{m}$ diameter beads into the pumping liquid (water) and observing the time taken by the particle to travel a fixed distance. In order to eliminate pressure effects a channel design as shown in figure 4.a. was used. The system is filled with the pumping liquid with particles and then the outlet ports are sealed to eliminate pressure effects. The liquid flows in a circular fashion as shown in the picture and by observing the path traced by the particle flow rate is calculated for different applied voltage and pulse frequency. A second experiment was performed to calculate pumping pressure. The channels were redesigned to allow for a column of air to be trapped as shown in figure 4.b. The column is placed after the diffuser and the compression of air in the column due to pumping pressure for an applied voltage and different pulse frequencies is calculated.

(a)

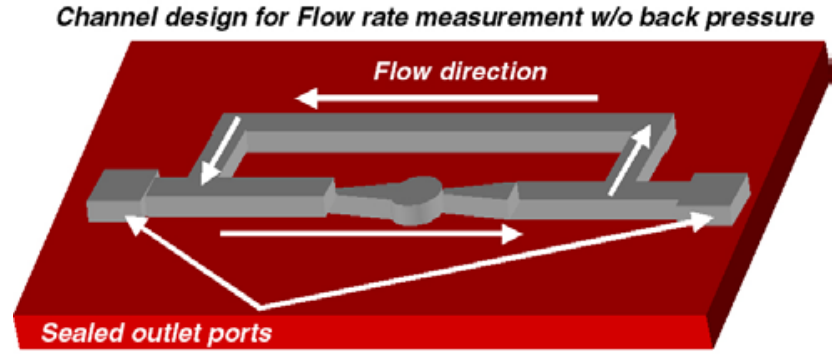

(b)

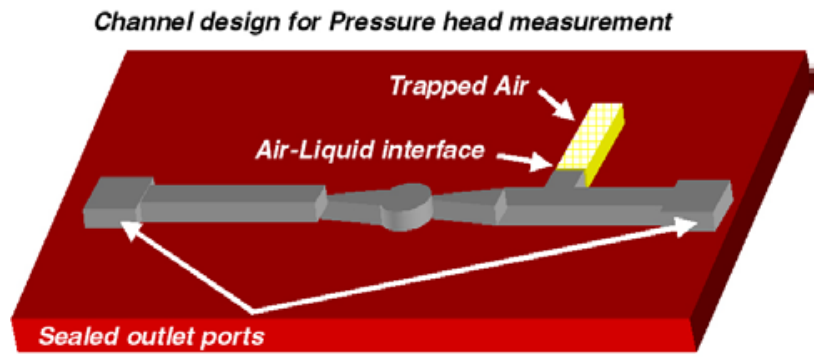

Figure 4. Channel design for calculation of (a) flow rate with zero back pressure and (b) pumping pressure.

\section{RESULTS AND DISCUSSION}

The surface profiles obtained due to deflection of the membrane on expansion of wax at different applied voltages is shown in figure 5 . Voltages up to $5 \mathrm{~V}$ were applied and a maximum deflection of $25 \mu \mathrm{m}$ was obtained at $5 \mathrm{~V}$. The deflecting $4 \mu \mathrm{m}$ parylene $\mathrm{C}$ membrane containing the wax failed at higher voltages due to high stress produced on wax expansion. 
Deflection heights at different applied voltages

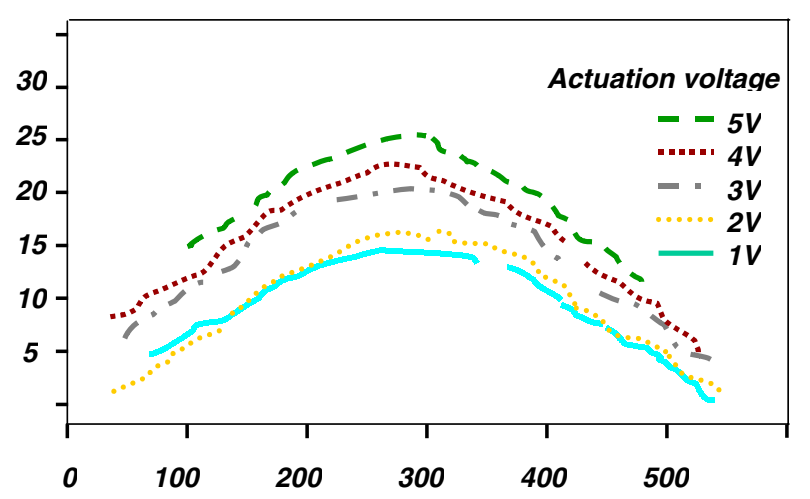

Deflection height $(\mu \mathrm{m})$ vs. Distance $(\mu \mathrm{m})$

Figure 5. Optical profiles of the deflection heights of the wax actuator surface obtained at different voltages using a ZYGO optical surface profilometer.

Flow rate measurements were done using the setup as shown in figure 4(a) and the particle flow velocities were measured for different applied voltages and pulse frequencies. Volume flow rates were calculated and plotted (figure 6) and maximum flow rates of $70 \mathrm{nl} / \mathrm{min}$ was obtained at an applied voltage of $5 \mathrm{~V}$ and pulse frequency of $1 \mathrm{~Hz}$. The wax-based actuator operates best at lower frequencies $(0.5 \mathrm{~Hz}-1.5 \mathrm{~Hz})$. This is due to the slow response time of the carbowax. High frequencies result in low volumetric expansion and hence low volume displacement because the heat generated due to the pulse is not sufficient to melt the entire wax patch.

Experiments were performed to calculate the pumping pressure that can be generated using the nozzle-diffuser pump. The setup used was as shown in figure 4(b). The channels were filled with fluid and air was trapped in a column, which is located just past the diffuser. The pump was operated at $5 \mathrm{~V}$ and the pulse frequency was varied. The pressure generated was calculated from the data obtained for height of the trapped air column. Maximum pumping pressure of $1400 \mathrm{~Pa}$ was obtained at a frequency of $1 \mathrm{~Hz}$. Results are plotted in figure 7.

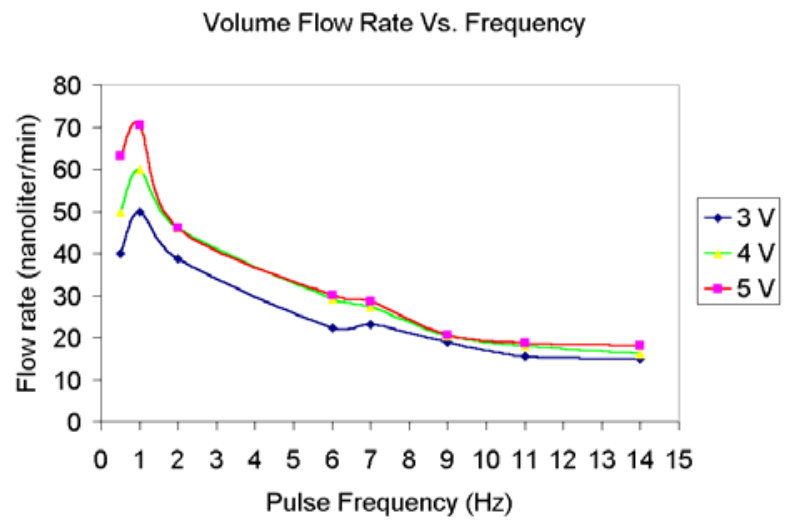

Figure 6. Volume flow rates obtained at different pulse frequencies for different applied voltages

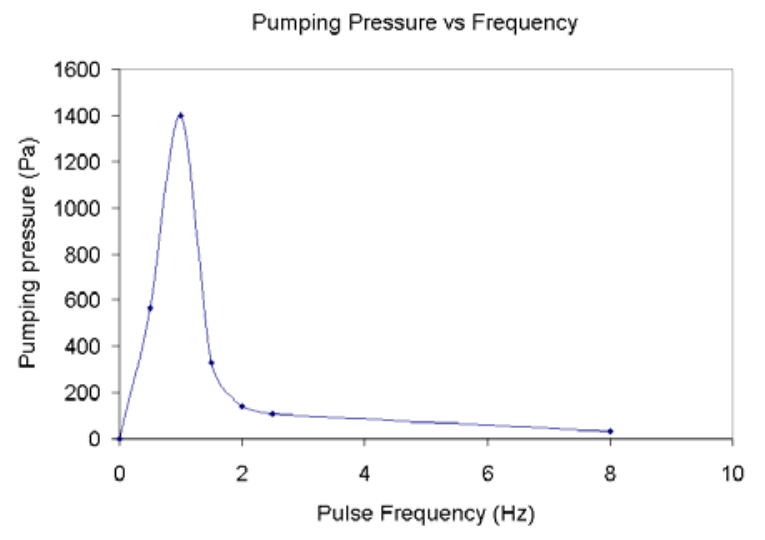

Figure 7. Pumping pressure generated at $5 \mathrm{~V}$ plotted for different pulse frequencies

\section{CONCLUSIONS}

We have developed a simple technique for fabrication and integration of low cost actuators within a disposable plastic microfluidic system for nozzle diffuser pumps. The pump operates at relatively low power and has maximum flow rates of about 70 $\mathrm{nl} / \mathrm{min}$ and pumping pressures of $1400 \mathrm{~Pa}$, which makes it attractive for applications like fluid transport, cooling and mixing in microfluidic systems.

\section{ACKNOWLEDGEMENTS}

This research work was supported by DARPA MEMS program under the contract F30602-98-2-0197.

\section{REFERENCES}

1. M. A. Burns, et. al., "An integrated nanoliter DNA analysis device", Science, 282, 253-258, (1998).

2. E. Kim, et. al., "Polymer microstructures formed by molding in capillaries", Nature, 376, 347, (1996).

3. P. Sethu, et. al., "Fabrication of Genetic Analysis Microsystems using Plastic Microcasting", Technical Digest of the 2000 SolidState Sensor and Actuator Workshop, Hilton Head Isl., SC, pp. 175 $-179,(2000)$.

4. E. T. Carlen and C. H. Mastrangelo, "Simple, High Actuation Power, Thermally Activated Paraffin Microactuator", International conference on Solid-State Sensors and Actuators (Transducers 99), Japan, 2, 1364-1367, (1999).

5. E. Stemme and G. Stemme, “A Valveless Diffuser/Nozzle based Fluid Pump”, Sensors and Actuators, A339, pp. 159-167. (1993).

6. Olsson, et. al., "Valve-Less Planar Pumps fabricated using thermoplastic replication", Technical Digest of the $10^{\text {th }}$ annual workshop of Micro Electro Mechanical Systems, Nagoya, Japan, pp. $305-310$, (1997). 